\title{
Auditoría administrativa y de comunicación organizacional: el caso del Programa de Telemedicina en México
}

\author{
Janet García González \\ (Cuernavaca, México) \\ Instituto Nacional de Salud Pública \\ Ingrid Karina Rodas Castillejos \\ (Puebla, México) \\ Benemérita Universidad Autónoma de Puebla
}

\section{Palabras clave \\ comunicación organizacional telemedicina auditoría}

Resumen

\begin{abstract}
En respuesta al avance de la tecnología en salud se encuentra la telemedicina que representa la salud a distancia. Sin embargo, las prácticas administrativas y de comunicación son parte esencial de la implementación y operación del programa de telemedicina en México, concretamente en los servicios de salud del Estado de Puebla. Este artículo está dedicado a la descripción integral del programa, utilizando un modelo de auditoría administrativa, y aplicado a la situación actual de la administración y comunicación organizacional.
\end{abstract}

Financiación: Gobierno del Estado de Puebla, México. Programa Estatal de Telemedicina a través de los Servicios de Salud del Estado de Puebla (SSEP). Conflicto de intereses: no existe ningún conflicto de intereses ni sesgos a partir del financiamiento brindado por el Gobierno del Estado de Puebla, México, para la publicación de los resultados obtenidos en esta investigación. Contacto para correspondencia: Janet García González. Teléfono. +52 (777) 32930 


\section{Administrative and Organizational Communication Audit: the Case of the Telemedicine Program in Mexico}

\begin{tabular}{c}
\hline Keywords \\
\hline organizational communi- \\
cation \\
telemedicine \\
managerial audit
\end{tabular}

\section{Cómo citar el artículo}

García, J. y Rodas, I. (2011). Auditoría administrativa y de comunicación organizacional: el caso del Programa de Telemedicina en México. Revista de Comunicación y Salud, 1(2), pp. 19-31.

DOI: http://doi.org/10.35669/revistadecomunicacionysalud.2011.1(2).19-31

\section{Introducción}

Las políticas sanitarias actuales consideran necesaria la implementación de las nuevas tecnologías de la información, como registros de salud electrónicos, y proveer sistemas de salud informatizados. La información administrativa es esencial para el desarrollo del sistema de salud, dada la naturaleza que tiene en el manejo de una gran cantidad de información. Para ello, se necesita integrar nuevas evidencias científicas a la práctica. Mientras que los beneficios de la tecnología en salud son claros en teoría, adaptar las nuevas tecnologías a los sistemas de salud ha sido difícil y el promedio de su uso ha sido limitado (Chaudhry, 2006: 10).

En la última década se ha comprobado un creciente interés en la informática, con recursos que pueden ser utilizados directamente por los pacientes. Un ejemplo de esto es la telemedicina. Los pacientes han tenido acceso a los hospitales a través de resultados médicos electrónicos (como el seguimiento a distancia utilizado para la captura de medidas específicas de enfermedad por vía electrónica, tales como glucosa en la sangre o los signos vitales), registros personales de salud (expediente clínico electrónico); y éstos se han utilizado directamente a través de videoconferencia sincrónica entre médico y paciente para la atención especializada de su salud (Shea, 2006). 
En la República Mexicana la cobertura de tecnologías de la información y comunicación es todavía limitada dentro del sistema de salud en relación con los requerimientos internacionales actuales. El programa de telemedicina que opera en el Sistema de Salud en México se implementó como parte de un proyecto de desarrollo tecnológico. La enorme demanda que exige la salud a distancia puede pasar de ser un reto a una oportunidad si se considera un análisis tanto administrativo como de comunicación, en beneficio de los grupos vulnerables que requieren de la atención de su salud a través de la telemedicina.

Un programa de telemedicina requiere tener como visión la innovación y la competitividad. Los cambios que exige la globalización no dependen solamente de conocerla internamente, sino también de aceptar que se cuenta con barreras externas que impiden el cumplimiento de sus metas (Koontz, 2004). Por lo tanto, un programa de esta naturaleza tiene la obligación de no descuidar el entorno interno y externo en el que se desenvuelve, debido a que estos elementos son determinantes para su desarrollo. Es importante que al analizar el proceso administrativo se destaque la comunicación porque contribuye a la estabilidad y al cumplimiento y seguimiento de objetivos.

La comunicación, en su parte organizativa, justifica la manera de dar a conocer los objetivos del programa y el seguimiento de metas, involucrando tanto a los que manejan el programa internamente como a los usuarios, es decir, su público externo. Desde el punto de vista de la comunicación organizativa estos conceptos deben incluirse en la parte de las previsiones. Con ello define el tipo de comunicación interna y externa que se desarrolla en la organización. Al igual, define la filosofía, estrategias y actividades que buscan cumplir con los objetivos del programa de telemedicina.

Por lo tanto, para la evaluación del programa de telemedicina se utilizó la auditoría administrativa y de comunicación, tomando como estudio de caso el Estado de Puebla, México. Para ello, se analizaron los elementos del campo administrativo, organizativo y de comunicación en las que se encuentra. Contar con un análisis integral de la administración del programa determina las estrategias, diseño, aplicación y alcance de los elementos que integran el programa.

\subsection{Hacia la problemática del Programa de Telemedicina ${ }^{1}$}

La utilidad y la importancia de la telemedicina son cada vez más evidentes. La disminución de los tiempos de atención, diagnósticos y tratamientos más oportunos, la mejora en la calidad del servicio, la reducción de costos de transporte, la atención continuada, los tratamientos más apropiados, la disminución de riesgos profesionales, la posibilidad de interconsulta y una mayor cobertura son, entre otras, algunas de sus ventajas. La telemedicina se logra con la integración de la medicina, las telecomunicaciones, tecnologías de información y la ingeniería biomédica. Se considera una herramienta tecnológica para el intercambio de imágenes, voz, datos y vídeo

\footnotetext{
${ }^{1}$ El proyecto de telemedicina llegó a México en el año 1996, bajo el concepto de "Telesalud". Se establece formalmente en su Estrategia No. 10 del Programa Nacional de Salud, que establece fortalecer la inversión en recursos humanos, investigación e infraestructura en salud; y particularmente en las siguientes líneas de acción: telemedicina, invertir en redes; utilizar los avances del internet; y el proyecto e-Salud para mejorar y hacer más eficiente la práctica médica. Estos lineamientos dan la pauta para el diseño, instrumentación y operación del Programa de Acción e-Salud
} 
por algún medio electrónico, que permite el diagnóstico y la opinión de casos clínicos.

Con la incorporación de estas nuevas tecnologías a los servicios de salud se abre la posibilidad de que, mediante un ejercicio de equidad, la población cuente con servicios de salud esenciales y con mayor acceso a la atención especializada, acercando estos beneficios a las zonas rurales y pueblos indígenas, y dando impulso a su distribución homogénea entre toda la población.

No se trata sólo de incorporar tecnologías novedosas. El objetivo es generar alternativas de valor apoyándose en un sistema tecnológico de contenido social, que ofrezca las herramientas y oportunidades que hoy en día es posible alcanzar mediante la interrelación de la salud con las tecnologías de la información y las telecomunicaciones. Además se logra que el Estado sea un regulador y promotor, ampliando el acceso de los habitantes a los servicios y al mundo globalizado; esto le exige atender aspectos tecnológicos.

El seguimiento bajo métodos administrativos puede alertar al personal del proyecto acerca de los problemas que presenta el programa, al dar una explicación sistemática acerca de si se efectúa o no el programa, si opera de acuerdo con el diseño y si alcanza a la población objetivo.

El programa de telemedicina en los Servicios de Salud del Estado de Puebla (México) fue implementado desde el año 2000. Sin embargo, en la actualidad el programa no se ha evaluado de ninguna forma; esto implica el desconocimiento de su desarrollo y de los beneficios que propiamente trae consigo la telemedicina. La naturaleza del departamento encargado del programa (informática) hace que sus enfoques sean totalmente tecnológicos, para lo cual ha desarrollado actividades que cubren este aspecto, teniendo como resultado una discrepancia entre las condiciones actuales y las especificadas por las metas del programa.

La debilidad de la implementación del programa de telemedicina es no referir aspectos administrativos y menos de comunicación que contribuyan a alcanzar las metas establecidas. No existe una planeación y se establecen las acciones de acuerdo a la experiencia. Por tal razón, más que tratar los problemas según se presente el caso, se requiere una evaluación específica de la administración y la comunicación. Además, los resultados de la evaluación se conciben como una información esencial para apoyar a los diseñadores del programa y a los directivos sobre los resultados de las condiciones existentes del programa. Con esto es posible hacer modificaciones o rediseño del programa.

Por otro lado, la institución sólo considera como evaluación el cumplimiento de indicadores sin tomar en cuenta los medios utilizados para llegar a ellos. El impacto que representa para los usuarios y prestadores de los servicios de salud no coincide con el bajo financiamiento y control administrativo. Esto limita su expansión a más municipios de la región. Ante esta situación la presente investigación plantea indagar la administración integral y la comunicación organizacional del programa de telemedicina en los Servicios de Salud del Estado de Puebla bajo una propuesta de modelo de auditoría. 
Este documento pretende, por una parte, describir las condiciones administrativas integrales del programa; por otra, incluir las condiciones de comunicación organizativa a partir del análisis de resultados del FODA ${ }^{2}$ con base en el análisis del informe derivado de la auditoría administrativa.

\subsection{Hacia una auditoría administrativa y de comunicación organizacional en el Programa de Telemedicina}

La administración dentro de una organización formal busca el cumplimiento de metas y objetivos. Para ello requiere que el personal sea el adecuado al puesto a desempeñar, y además que tenga conciencia de la importancia de las funciones que desarrollan los públicos internos y externos. Existen diversos elementos que integran el proceso administrativo. Para efectos prácticos de esta investigación se utilizó el enfoque de Agramante (1978) que divide al proceso en los siguientes: planeación, organización, interacción, dirección y control.

Para las fases del proceso administrativo la comunicación es esencial por varias razones, entre ellas, integrar las funciones administrativas. Un ejemplo de ello es la etapa de planeación donde los objetivos se comunican internamente en la estructura organizativa ${ }^{3}$. Otro ejemplo es la etapa de integración, donde la comunicación es esencial en los procesos de selección, evaluación y capacitación del personal. De igual modo, hace eficaz el liderazgo y la creación de un entorno conducente a la motivación para lograr que el desempeño responda a lo planeado. Por otra parte, el propósito del sistema de comunicación es enlazar a la organización con su ambiente externo, identificando las necesidades de los usuarios.

Debido a que la comunicación es analizada y definida desde diversas perspectivas, existen heterogéneas acepciones. Sin embargo, un elemento común que las integra es ubicar a la comunicación como parte integral del ser humano y, por ende, de las organizaciones. Además de esto, a través de la interacción se comparten múltiples significados y símbolos, provocando ciertos efectos en los interlocutores, como lo son los médicos, los pacientes y el personal operativo del programa.

Por otro lado, la evaluación del proceso administrativo y de comunicación del programa contribuye en la toma de decisiones, planeación operativa del programa y constituye la base de su desarrollo. Además, puede alertar al personal del proyecto acerca de los problemas que presenta el programa, si opera de acuerdo con el diseño y si alcanza a la población objetivo.

Los problemas administrativos dieron lugar a nuevas dimensiones en el pensamiento administrativo, analizando problemas cotidianos desde la planeación, organización, recursos humanos, materiales, tecnológicos y producción. En la búsqueda sistemática de soluciones se utiliza la ciencia

\footnotetext{
${ }^{2}$ FODA (SOWT en inglés) es una técnica que permite analizar la situación actual de una organización, estructura o persona, con el fin de obtener conclusiones que permitan superar esa situación en el futuro. Esta técnica permite el análisis de problemas en el contexto de la planeación y estrategia de las organizaciones. (Ansoff, 1965).

${ }^{3}$ Comunicación interna, definida como el modelo de mensajes compartidos entre los miembros de la organización, es la interacción humana que ocurre dentro de las organizaciones y entre los miembros de las mismas (Kreps, 1990).
} 
y la metodología científica dando lugar a una orientación técnica conocida como la auditoría administrativa 4 .

Existen diversos marcos de referencia para la auditoría administrativa. No obstante, en esta investigación se tomó el método de Rodríguez (1986), que consiste en proporcionar un panorama general, señalando el grado de efectividad con que opera cada una de las unidades administrativas que la integran. A continuación se desarrollan las etapas para su aplicación:

a) Estudio preliminar. Percibe información documental (reglamentos internos, informes, estadísticas, estados contables, presupuestos, contratos, entre otros); información sobre el campo de trabajo (organigramas, lista de funciones, datos sobre volúmenes de trabajo, examen de las condiciones en que se trabaja, formas y reportes utilizados); entrevistas (personas directamente relacionadas con la empresa para plantear problemas, descubrir funciones o departamentos que están originando algunas dificultades); y definición del área por investigar.

b) Planeación de la auditoría administrativa. Comprende la tarea de integrar un número de elementos que el auditor administrativo debe tener presente aún durante el análisis preliminar.

c) Investigación y examen de los elementos: funcional, procesal, analítico y ambiental. Mediante entrevistas, listas de comprobación, cuestionarios, observación de diagramas de flujo, manuales y organigramas, entre otros. El auditor deberá cerciorarse de obtener la información correcta y suficiente para dar un apoyo total a todo el análisis.

d) Análisis y evaluación de la información obtenida. La finalidad del análisis es preparar una síntesis de los resultados del proceso administrativo.

e) Informe de auditoría administrativa.

\section{Metodología.}

El objetivo general es describir la administración del Programa de Telemedicina en los Servicios de Salud del Estado de Puebla, México, a partir las condiciones administrativas, contables y de comunicación del programa, y de los resultados de la auditoría administrativa utilizando el análisis FODA.

\footnotetext{
${ }^{4}$ La auditoría administrativa es un examen comprensivo y constructivo de la estructura de una empresa, de una institución o de cualquier parte de un organismo, en cuanto a los planes y objetivos, sus métodos y controles, su forma de operación y sus equipos humanos y físicos (Leonard, 1991).
} 
Tabla 1. Identificación de variables e indicadores de la auditoría administrativa

\begin{tabular}{|ll|}
\hline Variables & Indicadores \\
\hline Planeación & Tipos de planes \\
\hline Organización & Estructura Organizacional \\
\hline Integración & Recursos Humanos \\
\hline Dirección & Comunicación \\
\hline Control & Evaluación \\
\hline
\end{tabular}

Fuente: elaboración propia

Dado lo anterior, el estudio corresponde a un estudio tipo descriptivo, observacional y de tipo transversal, retrospectivo y unicéntrico.

El estudio está dividido en tres etapas:

1) Metodología cualitativa. Se realizó entrevista estructurada al personal encargado del Programa de Telemedicina a nivel estatal y a nivel directivo del Hospital del Municipio de Zacatlán.

2) Metodología cuantitativa. Instrumento de medición de la auditoría administrativa a través de un cuestionario estructurado con reactivos de tipo alternativo-nominal (ver tabla 1). Universo de trabajo: médicos de consulta externa adscritos al Hospital de Zacatlán de los Servicios de Salud del Estado de Puebla, México.

3) Análisis de resultados a través del FODA. Fundamentándose en el análisis de los resultados la auditoría administrativa del ambiente externo e interno.

\section{Resultados}

\subsection{Primera etapa.}

Las entrevistas estructuradas realizadas a los directivos del programa de telemedicina arrojaron que ellos determinan la planeación como la parte importante del proceso administrativo, en donde el personal a cargo del programa debe conocer e identificarse con la misión de éste para el cumplimiento de sus metas. El conocimiento de esta filosofía no se identifica entre el personal como lo plantea el programa nacional, únicamente lo relacionan con el beneficio que tiene el programa. Como consecuencia, existe una diferencia entre lo que se plantea a nivel federal y lo que en realidad se conoce del programa.

Este programa carece de un organigrama importante para determinar la etapa de integración de personal para el desarrollo de sus actividades en las consultas de telemedicina. Así, los médicos lo consideran una actividad extra y hace más complejo la aceptación del programa. Por otro lado, el personal se encuentra desvinculado con el programa. Esto se debe a que la capacitación se centra en desarrollar un carácter de uso de tecnologías de la información sin tomar en cuenta la inducción del puesto que familiariza al individuo con sus actividades. 
Para la dirección se analizó el tipo de comunicación que se maneja en el programa, desde los canales de comunicación que integran las funciones administrativas hasta el sistema de comunicación que se establece con su ambiente externo. La comunicación en el programa se desarrolló sólo a nivel jerárquico, es decir, con comunicación vertical unidireccional y es la forma como se presentó el programa al personal, sin una difusión e información adecuada. Esto determinó la nula aceptación y la inoperatividad del programa.

Otro elemento importante es el control que implica la medición del desempeño y corrección de desviaciones con respecto a los planes. El programa de telemedicina no cuenta con ningún tipo de evaluación. Los resultados que se tienen del programa son indicadores estadísticos.

\subsection{Segunda etapa.}

Los resultados estadísticos se exponen de acuerdo a los cinco pasos del proceso administrativo, derivado del cuestionario aplicado a los médicos.

Resultados de planeación. Se carece de conocimiento claro de los objetivos del programa entre el personal médico y de los directivos del hospital y del departamento que controla a nivel estatal el programa. El $25 \%$ de todos ellos conoce el objetivo de su trabajo. Más del $90 \%$ opina que no existen políticas, estrategias y planes para llevar a cabo el programa y poder dar cumplimiento a sus metas. Sólo el $31 \%$ conoce el procedimiento para ejecutar el programa. Por otro lado, el $69 \%$ lo desconoce por completo y el $6 \%$ tiene información de él a través de medios audiovisuales, escritos o gráficos.

Resultados de organización. No existe una adecuada organización dado que no cuenta con un manual de organización del personal para telemedicina; el 94\% opina que no existe este diseño del puesto y que al igual desconoce las funciones y el proceso para ejecutar el programa de telemedicina. La mayoría (87\%) considera que los puestos no son suficientes.

Resultados de integración. No se cuenta con una inducción del programa hacia el personal y mucho menos a los usuarios. En cuanto a la evaluación del personal no se incluye el desempeño del personal en las funciones de telemedicina.

Resultados de dirección. Se detectaron factores de deficiencia: inexistencia de grupos de trabajo como comités para el programa de telemedicina e inadecuados medios de comunicación internos y externos para la difusión del programa. Todos estos con un porcentaje del $94 \%$ opinando negativamente y $6 \%$ consideró positivamente estas características.

El $62 \%$ conoce las vías de comunicación que se utilizan en el hospital entre los trabajadores y los directivos. La mayoría (87\%) considera que ha utilizado estos medios de comunicación para tratar aspectos sobre telemedicina además de considerar adecuados los procesos de comunicación en general dentro de la institución. Por último, el $62 \%$ del personal considera que existen desafíos de comunicación externa a los que se enfrenta actualmente el programa de telemedicina. 
Resultados de control. El personal desconoce (94\%) algún tipo de control sobre los resultados del programa y el $38 \%$ desconoce las normas del programa. Sin embargo, para fines de estadística sobre el control del programa, existe un documento interno para conocer las consultas diarias de telemedicina en los hospitales. A pesar de ello no se considera oficial debido a la carencia de un presupuesto designado para su operación.

La etapa de control demuestra ser de los porcentajes más deficientes en los factores evaluados, a pesar de ser considerado dentro del proceso administrativo como prioritario para desarrollo del programa de telemedicina.

\subsection{Tercera etapa.}

Tabla 2. Resultados del FODA derivados de la Auditoria Administrativa

\begin{tabular}{|c|c|c|}
\hline Factores internosifactores externos & $\begin{array}{l}\text { Fortalezas } \\
\text { F1 Programa operativo con misión, } \\
\text { visión y objetivos. } \\
\text { F2 Referencia de manual operativo } \\
\text { donde se contemplan las } \\
\text { actividades, funciones y perfiles del } \\
\text { personal } \\
\text { F3 Experiencia en el uso de la } \\
\text { telemedicina en el hospital } \\
\text { F4 Programa de acción con } \\
\text { estrategias específicas para la } \\
\text { implementación del programa } \\
\text { F5 Registro estadistico del programa } \\
\text { que contempla el número de } \\
\text { consultas } \\
\text { F6 Programa de acción con } \\
\text { indicadores junto con su fómula } \\
\text { para la medición del programa } \\
\text { F7 Presentación del programa en el } \\
\text { hospital en las reuniones del comité } \\
\text { como medio de comunicación entre } \\
\text { el personaly el hospital }\end{array}$ & $\begin{array}{l}\text { Debilidades } \\
\text { D1 Desconocimiento de la misión y } \\
\text { visión del programa de acción } \\
\text { telemedicina por directivos y } \\
\text { operativos del hospital } \\
\text { D2 Indefinición de las funciones del } \\
\text { programa, el personal las desconoce } \\
\text { y el área directiva no las puede } \\
\text { implementar correctamente } \\
\text { D3 Operación del programa con } \\
\text { mayor orientación tecnológica } \\
\text { D4 Carencia de políticas propias de } \\
\text { la institución para operar el programa } \\
\text { D5 Sin procedimientos ni estrategias } \\
\text { específicos para implementar el } \\
\text { programa parar insuficiente para } \\
\text { D6 Personal ín } \\
\text { atender el módulo de telemedicina } \\
\text { en el hospital. } \\
\text { D7 Sin evaluaciones que se apeguen } \\
\text { a los estándares del programa de } \\
\text { acción. }\end{array}$ \\
\hline $\begin{array}{l}\text { Oportunidades } \\
\text { 01 Presupuesto considerado por el } \\
\text { Departamento de Informática } \\
\text { O2 Personal médico apoya el } \\
\text { avance tecnológico para } \\
\text { capacitación y atención a la salud } \\
\text { O3 Conexión actual con el Hospital } \\
\text { General de Puebla para el uso de la } \\
\text { telemedicina } \\
\text { O4 Comunicación directa entre el } \\
\text { hospital y nivel central para asuntos } \\
\text { de la telemedicina }\end{array}$ & $\begin{array}{l}\text {-Desarrollo del plan de acción para el } \\
\text { programa } \\
\text {-Fomento del programa de acción } \\
\text {-Análisis y redefinición del manual } \\
\text { operativo } \\
\text { - Integrar los procedimientos } \\
\text {-Diseño de la infomación para la } \\
\text { capacitación } \\
\text {-Definición de los medios de } \\
\text { comunicación para el fomento y } \\
\text { desarrollo del programa } \\
\text {-Consolidar acciones con Hospital } \\
\text { General para la continuidad del } \\
\text { programa }\end{array}$ & $\begin{array}{l}\text {-Desarrollo de las políticas de } \\
\text { operación } \\
\text {-Desarrollo de las estrategias de } \\
\text { operación } \\
\text {-Definición de las funciones del } \\
\text { programa para las unidades } \\
\text {-Definición de datos estadísticos } \\
\text {-Definición del perfil de puestos } \\
\text {-Creación de modelos de evaluación } \\
\text { para el control del programa }\end{array}$ \\
\hline $\begin{array}{l}\text { Amenazas } \\
\text { A1 Sin coordinación propia para el } \\
\text { programa, a nivel central de la } \\
\text { institución } \\
\text { A2 Conexión y redes dependiente de } \\
\text { otra institución } \\
\text { A3 Sin presupuesto propio para el } \\
\text { programa desde nivel federal } \\
\text { A4 Personal con infomación } \\
\text { insuficiente y correcta del programa } \\
\text { A5 Usuarios con preferencia a la } \\
\text { forma tradicional médico-paciente }\end{array}$ & $\begin{array}{l}\text {-Impulsar la creación de una } \\
\text { coordinación } \\
\text {-Consolidar convenios para equipos } \\
\text { y servicios de red } \\
\text {-Consolidar presupuesto a nivel } \\
\text { federal } \\
\text {-Optimizar instalaciones eliminando } \\
\text { obstáculos a innovaciones } \\
\text { tecnológicas }\end{array}$ & $\begin{array}{l}\text {-Establecer canales de comunicación } \\
\text { externos } \\
\text {-Evaluar la opinión del usuario } \\
\text {-Promoción de un programa de } \\
\text { orientación para la utilización de la } \\
\text { telemedicina con énfasis en } \\
\text { relaciones médico paciente } \\
\text {-Diseño de la información para el } \\
\text { conocimiento del programa }\end{array}$ \\
\hline
\end{tabular}

Fuente: elaboración propia 
A partir de los resultados del FODA, se puede afirmar que la misión y la visión del programa de telemedicina es poco conocida por parte del personal médico que labora en el hospital, el conocimiento sobre el programa se ha realizado a partir de la capacitación que otorgó el Departamento de Informática. Los objetivos, políticas y estrategias para llevarlo a cabo se basan en las necesidades de tecnología y de redes del Departamento de Informática sin estar orientadas a los objetivos propios del programa operativo federal, y éstos son desconocidos por el personal. Y los que tienen alguna idea de ello concuerdan que los objetivos principales son llevar la salud a las zonas marginadas para evitar el gasto que le implica al paciente viajar a la zona centro del Estado, y, por otro lado, contar con la ayuda de especialistas para auxiliar en el diagnóstico de enfermedades.

La planeación de las actividades para el programa es elaborada por el director del hospital, persona encargada de determinar los horarios en los que se dará la consulta de telemedicina y la programación para los pacientes. Estos planes se van modificando de acuerdo con las necesidades que se presenten, como por ejemplo horarios no disponibles, falta de personal médico, falta de conexión de la red de telecomunicación... Las estrategias del programa están planteadas en el programa de acción "E-Salud Telemedicina" de la Secretaría de Salud, pero actualmente no se cumplen por falta de presupuesto y apoyo de tecnología para el programa.

La estructura organizativa de la institución maneja una departamentalización matricial (horizontal con sistemas verticales) ya que está integrada por departamentos que dan servicio a todos los niveles de la estructura organizacional. Esta estructura favorece la operación del programa de telemedicina, debido a que existe una coordinación médica a nivel horizontal, se puede implementar el programa a todos los departamentos que dependen de esta coordinación. El programa de telemedicina se coordina en el área de atención médica, y dado que el personal médico es de mayor plantilla para un hospital, la telemedicina depende en gran parte de la aceptación y ejecución del programa por parte del personal médico. El programa dispone de una descripción del puesto incompleta. Existe un documento que maneja solamente el Área de Informática, que menciona las características generales que el trabajador requiere para cubrir el puesto. Sin embargo, no se cuenta con una capacitación específica para éste.

En cuanto a la comunicación interna, el personal conoce los diferentes tipos que se utilizan dentro del hospital. Son los siguientes: formal, a través de juntas de comités y oficios relacionados con el tema; informal, cuando el personal realiza comentarios y expresa sus opiniones personales con sus compañeros de trabajo; imperativa, se desarrolla a partir del establecimiento horarios y fechas laborales específicas; exhortativa, se busca la participación del personal en diferentes actividades del hospital; vertical, el personal está informado a través de sus superiores; horizontal, se desenvuelve entre las diversas líneas jerárquicas al realizarse las juntas de trabajo. Sin embargo, la comunicación externa es deficiente en el soporte del programa, dado que no se cuenta con la información suficiente para conocimiento de los usuarios, generando con ello una imagen deficiente sobre el programa.

El control que se opera es únicamente desarrollado en reportes, éstos contemplan las metas programadas y las metas reales del programa sobre un tiempo determinado (mensual). En este mismo sentido, no hay control interno que contribuya a evaluar al personal médico. En 
cuanto al control externo, no se ha realizado una medición sobre la opinión del usuario ante el programa; consecuencia de ello es la falta de una estrategia de difusión de éste. El único mecanismo de control se basa en el número de consultas que se han dado mensualmente, sin desarrollar indicadores que pueden contribuir a la administración del programa.

El programa de telemedicina carece de una herramienta que permita identificar el camino a seguir para realizar las intervenciones, tanto con propósitos administrativos como de eficiencia, eficacia e impacto de los objetivos para lo que fue creado el programa. El costo del programa y las limitaciones de los recursos para operarlo, sobre todo de fondos públicos, genera desconfianza en su operatividad y en el apego a los requerimientos legales y operativos.

\section{Discusión y conclusiones}

Los resultados obtenidos pueden determinar las propuestas de mejora y de esta forma prevenir las posibles desviaciones de objetivos; llevar a cabo análisis y desarrollo de los costos del proyecto con énfasis en los beneficios de la salud de la población meta. Así como también el desarrollo de los planes de acción de comunicación organizacional enfocados en el conocimiento, aceptación y utilidad del programa de telemedicina.

Existen estudios piloto y experiencias de programas de telemedicina que se han desarrollado pero los evaluados son limitados. Sin embargo, dado los costos de los proyectos es necesario realizar un estudio adecuado de evaluación donde se especifique los beneficios y ventajas de su implantación. Esto permitiría orientar su desarrollo, promoción y seguridad a los usuarios sobre la eficiencia, eficacia y utilidad y con ello apoyar la viabilidad de desarrollo a este tipo de proyectos a medio y largo plazo.

Actualmente en cualquier organización es necesaria la visión de innovación y competitividad. Por lo tanto, está obligada a no descuidar el entorno tanto interno como externo en el que se desenvuelven debido a que son factores determinantes que contribuyen al desarrollo de ésta. En otras palabras, la organización debe periódicamente realizar revisiones y análisis de su administración para conocer el funcionamiento total de sus procesos, procedimientos y programas con el fin de detectar deficiencias que amenacen su estabilidad. A su vez, los resultados obtenidos pueden determinar las propuestas de mejora y de esta forma prevenir las posibles desviaciones de objetivos.

Los cambios que exige la globalización no dependen solamente de conocer la organización internamente sino también aceptar que se tienen limitaciones y errores que impiden un crecimiento sano en ella, pero también es importante conocer las fortalezas y oportunidades que pueda tener para analizarlas junto con su ambiente externo en que se desarrolla (debilidades y amenazas).

En el campo de la salud la evaluación puede ser tecnológica, económica o de sistemas de salud. Esta investigación propuso la evaluación administrativa integral y de comunicación con la finalidad de que se utilice como modelo de evaluación útil. 


\section{Referencias}

Agramante, Mier y Terán, Norberto. (1978). Curso de teoría de la administración. México: Ecasa.

Alvira Martín, Francisco. (1991). Metodología de la evaluación de programas. Madrid: Centro de Investigaciones Sociológicas.

Ansoff, Igor H. (1965). Corporate Strategy: an Analitytic Aproach for Growth and Expansion. New York: Mc Graw Hill.

Attkinsson, Clifford et. al. (1999). Administración de hospitales: fundamentos y evaluación del servicio hospitalario. México: Trillas.

Buch, Tomás. (1999). Sistemas tecnológicos. Buenos Aires: Aique.

Cervantes Abreu, Salvador. (1982). Dinámica de la auditoría administrativa. México: Ediciones contables y administrativas.

Chiavenato, Idalberto. (1986). Introducción a la teoría general de la administración. México: Mc Graw Hill.

Cohen, Ernesto et al. (2006). Evaluación de proyectos sociales. México: Siglo XXI Editores.

Chaudhry, B. et al. (2006). Systematic Review: Impact of Health Information Technology on Quality, Efficiency, and Costs of Medical Care. Annals of Internal Medicine. Vol. 144, n 10, pp. 742-752.

Fernández Arena, José Antonio. (1992). La auditoría administrativa. México: Diana.

Garrido, Francisco Javier. (2001). Comunicación estratégica. Barcelona: Gestión 2000.

Koontz, Harold, y Weihrich, Heinz. (2004). Administración, una perspectiva global. México: Mc Graw Hill.

Leonard, William P. (1991). Auditoría administrativa. México: Diana.

Lucas Marín, Antonio. (1997). La comunicación en la empresa y en las organizaciones. Barcelona: Bosch Casa Editores.

Reyes, Agustín. (1968). Administración moderna. México: Editorial Limusa.

Rodríguez Valencia, Joaquín. (1991). Sinopsis de la auditoría administrativa. México: Editorial Trillas.

Rossi, Peter H. y Freeman, Howard. (1989). Evaluación, enfoque sistemático para programas sociales. México: Editorial Trillas. 
Santillana, Juan Ramón. (1994). Conoce las auditorías. México: Ecasa Editores.

Shea, Steven; Weinstock, Ruth y Starren, Justin. (2006). Randomized Trial Comparing, Telemedicine Case Management. Journal of the American Medical Informatics. Vol. 13, $\mathrm{n}^{\circ}$ 1, pp. 40-51. doi: 10.1197/jamia.M1917

Solivérez, Carlos E. (1991). Ciencia, técnica y sociedad. Buenos Aires: Facultad Latinoamericana de Ciencias Sociales Editores.

Steiner, George. (1999). Introducción al pensamiento administrativo. México: Editorial Trillas.

Villafañe, Justo. (1998). Imagen positiva, gestión estratégica de la imagen de las empresas. Madrid: Pirámide.

W.AA. (2001). Dirección de comunicación empresarial e institucional. Barcelona: Gestión 2000. 